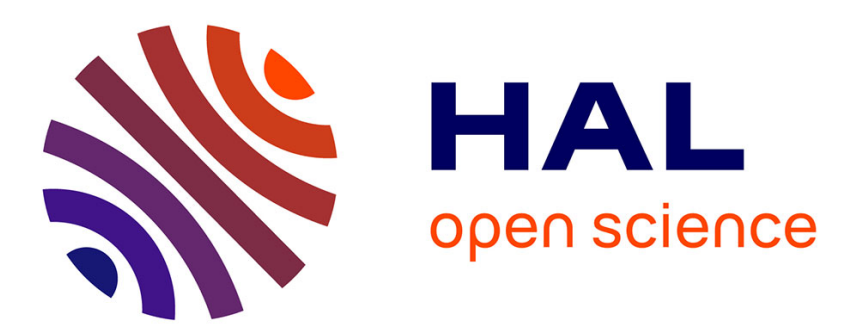

\title{
On the park effect in arrays of oscillating wave energy converters
}

\author{
Aurélien Babarit
}

\section{To cite this version:}

Aurélien Babarit. On the park effect in arrays of oscillating wave energy converters. Renewable Energy, 2013, 58, pp.68-78. 10.1016/j.renene.2013.03.008 . hal-01145167

\section{HAL Id: hal-01145167 \\ https://hal.science/hal-01145167}

Submitted on 4 Jun 2019

HAL is a multi-disciplinary open access archive for the deposit and dissemination of scientific research documents, whether they are published or not. The documents may come from teaching and research institutions in France or abroad, or from public or private research centers.
L'archive ouverte pluridisciplinaire HAL, est destinée au dépôt et à la diffusion de documents scientifiques de niveau recherche, publiés ou non, émanant des établissements d'enseignement et de recherche français ou étrangers, des laboratoires publics ou privés. 


\title{
On the park effect in arrays of oscillating wave energy converters
}

\author{
A. Babarit* \\ LHEEA Lab, LUNAM Université, Ecole Centrale de Nantes - CNRS, 1 rue de la Noe, 44300 Nantes Cedex 3, France
}

\begin{abstract}
This paper aims to provide guidelines for designing the layout of arrays of oscillating Wave Energy Converters (WECs) based on a review of the literature of wave interactions and park effect in WEC arrays that has been published over the past 30 years.

First, the fundamentals of wave energy absorption by oscillating bodies are summarised, and the principal differences between the park effect in arrays of wave energy converters and wind turbines are highlighted. Then, the numerical approaches commonly used to deal with WEC arrays are outlined briefly and their limitations are discussed. It is argued that, at present, only Boundary Element Methods (BEM) are capable of the appropriate analysis. Finally, previous work on wave interactions and park effect in WEC arrays is reviewed. Similar trends are found in these studies, which allow conclusions to be drawn regarding the significance of the park effect as a function of the number of WECs in the array and their spacing. Based on these conclusions, the following tentative guidelines are proposed:

For small arrays of conventional devices (fewer than 10 devices of typical dimension 10-20 m) with usual layouts (regular or shifted grids with separating distance of order 100-200 m), the park effect appears to be negligible. For larger arrays (more than 10 devices), a negative park effect seems to be increasingly important with increasing number of rows (the lines of WECs perpendicular to the incident wave direction). Therefore, the number of rows should remain as small as possible, with a separating distance as large as possible. For arrays of non-conventional WECs (WECs of typical dimensions much larger than $10-20 \mathrm{~m}$ ), no information has been found. However, trends similar to the previous cases could be expected, provided that aspect ratios are maintained.
\end{abstract}

\section{Introduction}

By definition, any renewable energy converter absorbs a certain amount of the energy resource. Therefore, the total available resource is reduced for other energy converters located nearby. In consequence, the total power from an array of $N$ energy converters is less than $N$ times the power of one isolated unit. This is called the park effect.

The park effect is well known in the wind turbine industry. Studies have shown that the park effect decreases as a function of increasing distance between the wind turbines. This has resulted in guidelines for the longitudinal and lateral distances between wind turbines.

There is also a park effect in wave energy conversion, which must be taken into account when designing an array of Wave Energy Converters (WECs). In contrast to the wind industry, there are

\footnotetext{
* Tel.: +33 240371623.

E-mail addresses: aurelien.babarit@ec-nantes.fr, ababarit@hotmail.fr.
}

no guidelines at present for their separating distance, although a large number of studies dealing with arrays of WECs has been published over the past 30 years. By reviewing this literature, the aim of the present paper is to provide a summary of the current knowledge on the park effect in arrays of WECs and to provide guidelines for its mitigation.

First, the fundamentals of wave energy absorption by oscillating bodies are summarised, and the principal differences between the park effect in arrays of wave energy converters and wind turbines are highlighted. Then, the numerical approaches commonly used to deal with WEC arrays are outlined briefly and their limitations are discussed. It is argued that, at present, only Boundary Element Methods (BEM) are capable of the appropriate analysis. Finally, previous work on wave interactions and park effect in WEC arrays is reviewed. Similar trends are found in these studies, which allow conclusions to be drawn regarding the significance of the park effect as a function of the number of WECs in the array and their spacing. Based on these conclusions, tentative guidelines are proposed for the design of the layout of WEC arrays to mitigate the park effect. 


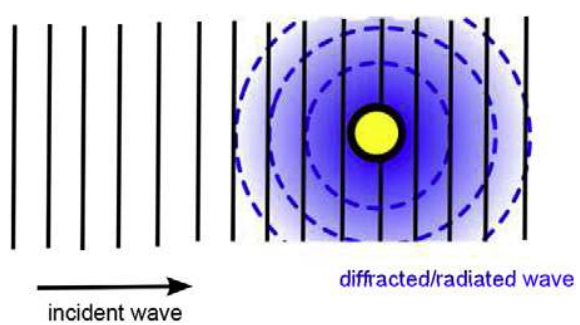

Fig. 1. Schematic of the wave pattern around an oscillating moving structure with incident waves.

\section{Wave interactions in arrays of wave energy converters}

\subsection{Radiated/diffracted wave field around an oscillating structure}

To understand the park effect in arrays of wave energy converters, it is necessary to understand the physical process of how a wave energy converter interacts and absorbs energy from the incident wave field.

The fundamental idea is that a fixed or moving structure in an incident wave field will give rise to additional wave systems known as diffracted or radiated waves (Fig. 1). ${ }^{1}$ They are governed by the same set of equations as the incident wave (Laplace equation in the fluid domain plus boundary conditions, in particular free surface conditions). They carry energy in the same way as the incident wave.

However, there is an important difference between the diffracted/radiated wave and the incident wave. An incident wave is a plane wave propagating in only one direction, whereas a diffracted/ radiated wave propagates in every direction from the source point (i.e. the oscillating structure). This is important to note because it means that one effect of the wave-structure interaction is to redistribute part of the incident energy, which had been propagating in a single direction, in all other directions. Therefore, the overall wave field is perturbed everywhere around the structure, and not only at the back (front and back being defined respectively to the direction of propagation of the incident wave).

\subsection{Wave energy absorption as an interference}

The principle of energy conservation implies that the energy in the diffracted/radiated wave is derived from the incident wave. This means that the phase in the diffracted/radiated wave is such that the incident wave is partly cancelled in some directions (which means that some energy is taken out), and partly increased in other directions (where some part of the energy is reemitted).

In the case of a WEC, in addition to energy being transferred from the incident wave to the diffracted/radiated wave, some of it is absorbed in what is referred to as a Power Take Off (PTO). In contrast to the case of zero wave energy absorption, effect of the PTO is observed as a change in the phase of the radiated wave, corresponding to an increase in incident wave cancellation and/or a decrease in incident wave amplification. Thus, by considering the total balance of energy, one would observe that some energy is missing in the fluid domain. This corresponds to the energy that has been absorbed by the WEC. This is explained by Ref. [1]:

\footnotetext{
1 Usually, linear theory is used to model wave-structure interaction. Thus, it is possible to separate the effects by applying the superposition principle. The diffracted wave corresponds with the waves generated by the fixed structure in response to the incident waves. The radiated wave corresponds with the waves generated by the moving structure in still water. The solution of the complete problem (moving structure in waves) is obtained by superimposing diffracted wave + radiated wave + incident wave.
}

"The physical law of conservation of energy requires that the energy-extracting device must interact with the waves such as to reduce the amount of wave energy that is otherwise present in the sea." or by Ref. [2]:

"To absorb energy from an incident wave, the radiated waves emitted from the surface(s) of an oscillating system must destructively interfere with the incident wave."

\subsection{A fundamental difference to wind energy: "wake" is meaningless for WECs}

A fundamental difference between wind energy and WECs is that in the latter, the perturbation of the wave field (diffracted/ radiated wave) is observed not only at the back, but at every location around the structure (Fig. 2).

In the case of wind energy, one can define the wake as the downstream region where the wind speed is modified. Only wind turbines located behind other wind turbines are affected. Conversely, wind turbines in the front of the wind farm are not affected by the wake of wind turbines located downstream.

In the case of wave energy, it is not appropriate to talk about wake because, as discussed above, the wave field is modified in every direction from the source. Therefore, every single WEC in the array interacts with all the others, whatever their locations. In particular, WECs located in the front row of the array (the ones which meet the incident wave first) are affected by WECs behind them. Their response to the waves (and thus their energy absorption) is different with and without the WECs behind. Indeed, several authors [3-5] have observed that the modification of the energy absorption due to the park effect could be of higher significance for the WEC in front than for the WEC behind. In several cases, it has been reported that the energy absorption is actually increased for the front row by the presence of a row behind. However, one should note that these reported increases are small (a few percent).

Therefore, it appears that, a priori, a WEC array must be considered as a whole when assessing the park effect.

\subsection{Decay of wave interaction with distance}

It follows from energy conservation that the energy in a radiated/diffracted wave propagating in direction $\theta$ decreases with increasing distance to the source. This has two remarkable consequences:

- It implies that if two WEC units are sufficiently far from each other, wave interaction effects can be neglected.

- Even directly behind a WEC, the unperturbed wave energy flux is recovered with distance: at large distances from the WEC, the wave energy resource is similar to what it would have been in the absence of the WEC.

In both cases, full recovery of the wave energy flux is achieved only when the distance reaches a sufficient magnitude, which is directly related to the intensity of the diffracted/radiated wave. One can expect that for bigger WECs, the perturbation will be stronger, and hence that larger distances will be required.

\section{Usual numerical approaches to deal with the park effect of WECs}

This section provides an overview of the available numerical tools. For a more comprehensive review of the strengths and weaknesses of these tools, see Ref. [6]. 

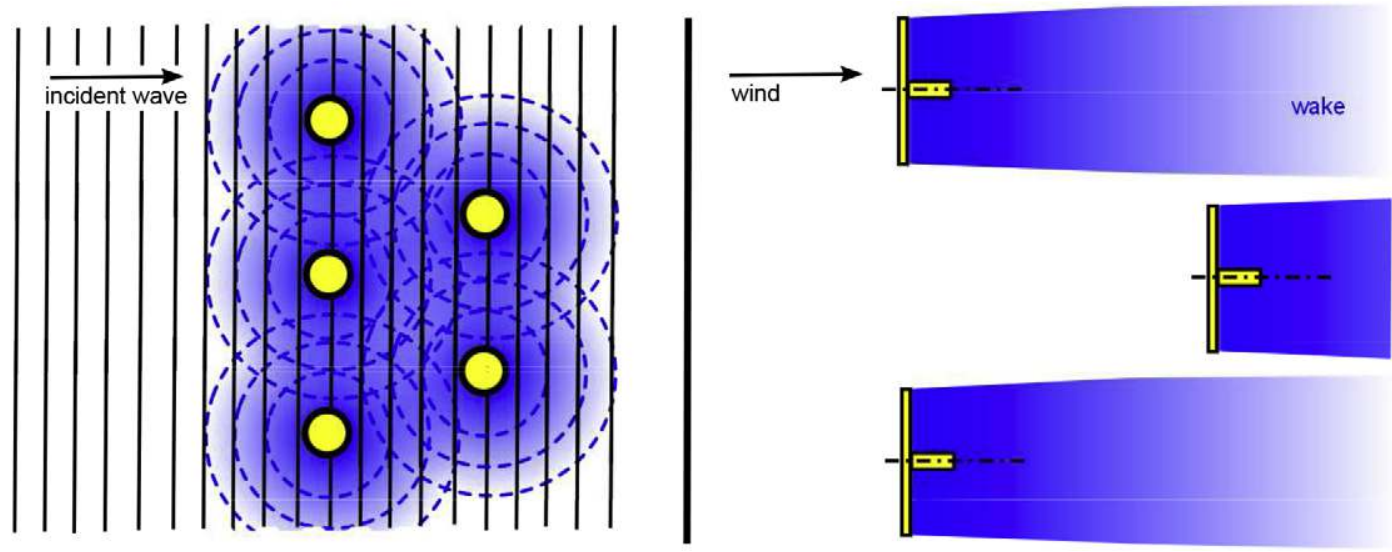

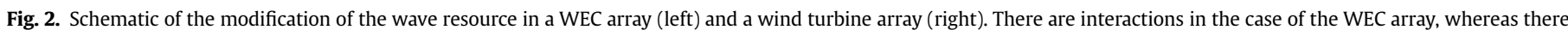
are not in the case of the wind turbine array.

\subsection{Analytical methods}

At the time of the early studies of the park effect in arrays of WECs [7-9], computing resources were limited. The methods used at that time were analytical studies of theoretical WECs (heaving axisymmetrical devices) that addressed only regular and unidirectional waves. Linear potential flow theory was used.

Analytical approaches are numerically efficient and are able to deliver interesting results even today, as in Ref. [3] or in Ref. [10]. However, their scope is limited, and they cannot be applied in practical cases of complex WECs with multiple Degrees of Freedom (DoF) or irregular waves.

\subsection{Boundary elements methods}

BEM-based numerical codes (WAMIT, ANSYS Aqwa, Aquaplus, ...) are, in theory, able to deal with any case of arrays without restriction, except the usual ones of linear potential theory (small amplitude of motions and wave steepness). An example of the results of a BEM calculation is shown in Fig. 3, taken from Ref. [5]. It shows the total wave field around an array of two clusters of eight floating Oscillating Surge Converters (OSWCs).

In practice, the memory and CPU requirements of these numerical models increase rapidly with the number of WECs in the array, and hence they are currently limited to relatively small arrays (typically 5 to 10 devices) [11-16]. Research to overcome the

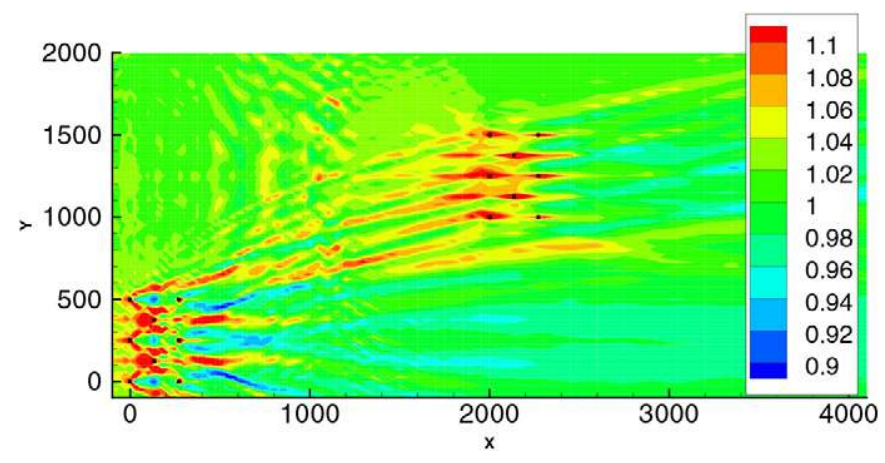

Fig. 3. Ratio of significant wave height of total wave field to the significant wave height in the incident wave for an array of two clusters of eight floating OSWCs (black dots). The $x$ and $y$ axis are the horizontal coordinates, in metres. The incident wave is unidirectional and irregular with $H_{s}=2$ metres, $T_{p}=8$ seconds. One can see that the wave field is modified at every location of the domain. Source: [5]. computing resource limitations that currently preclude the analysis of larger arrays is ongoing [17].

Another important limitation with this approach is that it assumes a uniform water depth whereas, in the case of a large array of WECs, bathymetry might vary significantly. In principle, this could be taken into account by meshing the sea bottom as well as the oscillating structures. However, it would lead to an enormous number of additional unknowns and computing resource would become, again, an issue.

\subsection{Asymptotic approximations}

With linear potential theory, one can show that the diffracted/radiated wave field is the sum of so-called near field and far field contributions [18]. The near field vanishes rapidly with distance to the body whereas the far field contribution decays much more slowly. Therefore, at some distance (which is usually not very large), only the far field contribution remains noticeable.

One can show [18] that the far field contribution can be described as a circular wave whose amplitude depends only on the direction of propagation $\theta$ :

$\eta_{\text {far field }}(r, \theta, t)=\Im\left(\frac{1}{\sqrt{r}} \tilde{H}(\theta) e^{i(k r-\omega t)}\right)$

where $(r, \theta)$ are the cylindrical coordinates, $\tilde{H}(\theta)$ is the so-called Kochin function which can be calculated using BEM codes [18], $\mathrm{k}$ and $\omega$ are the wavenumber and wave frequency, respectively.

Thus, there exists a semi-analytical representation for the radiated/diffracted wave at a sufficient distance from the structure. Locally, it can be further approximated as a plane wave of complex amplitude $1 / \sqrt{r_{0}} \tilde{H}\left(\theta_{0}\right)$, which allows wave interactions to be treated as if they were supplementary incident waves. The method, first proposed by Refs. [19], is called plane wave approximation. According to [11], the results of plane wave approximation have good agreement with BEM for sparse arrays.

\subsection{Boussinesq and spectral wave models}

These models and the set of equations on which they are based were initially developed to model wave propagation over large domains (a few kms for Boussinesq models, hundred of kms for spectral wave models). They are designed to be able to deal with bottom-induced effects (refraction, shoaling, white capping, 
etc. However, they are not well-adapted to dealing with moving structures such as a WEC or an array of WECs.

In these models, WECs are usually approximated as coefficients that represent partial energy absorption. In Refs. [20], they are modelled as sinks of constant strength regardless of wave frequency and direction, and their effect on the wave climate at the shore line is assessed using the SWAN model. Similar approaches can be found in Refs. [21-23], except that the numerical models are based on Boussinesq equations.

Constant strength sinks or transmission coefficients are poor representations of wave energy absorption by WECs because the power output of nearly all wave devices is dependent on the frequency of the incident wave conditions. Models employing coefficients that vary with frequency and direction have been proposed in Refs. [24,25].

These approaches are questionable. To date, none of these approaches is able to represent correctly the physical process of wave energy absorption by oscillating WECs. In particular, they disregard the fact that some of the energy is reflected in the direction opposite to the direction of propagation of the incident wave. This is illustrated in Fig. 4, taken from Refs. [25], in which one can see that the wave field at the front and sides of the WEC array is not modified. Moreover, the physical process by which wave energy is absorbed from the domain - i.e. interferences between the incident wave and the diffracted/radiated wave in which phase is essential - is not modelled. Furthermore, there is no clear protocol for calibrating the coefficients that represent the WECs.

For these reasons, results from studies based on these approaches are not taken into account in what follows.

\subsection{Combined used of asymptotic approach and Boussinesq or spectral wave models}

Although the direct use of Boussinesq or Spectral wave models for assessing the park effect in arrays of WECs is inadequate, it might be effective to combine these models with asymptotic approximations for the far field potential (which can be obtained using BEM). In other words, Boussinesq and/or spectral wave models could be used to compute the wave field at some distance from the WECs whereas the wave field close to the WEC would be

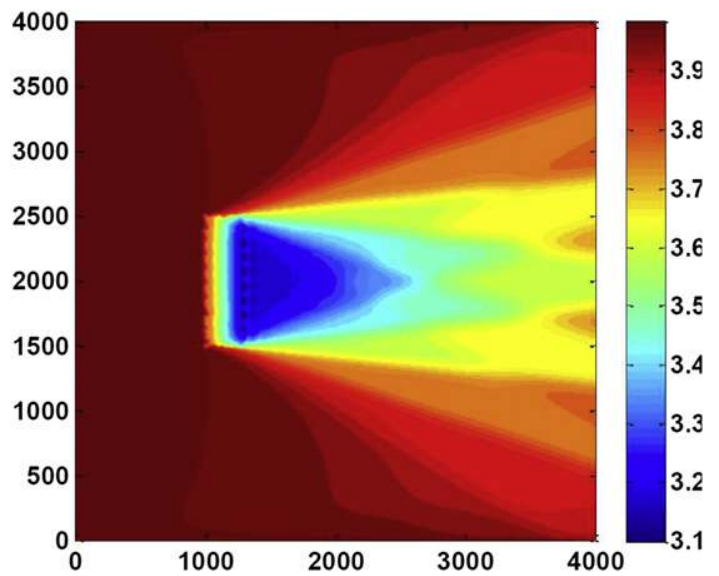

Fig. 4. Significant wave height $(\mathrm{m})$ for an array of WECs computed using TOMAWAC The $x$ and $y$ axis are the horizontal coordinates, in metres. The incident waves are irregular with directional spreading. Parameters are $T_{p}=10$ seconds, $H_{s}=4$ metres and spreading coefficient $s=5$. WECs are modelled as transmission coefficients depending on the direction and frequency. It is clear that, in contrast to Fig. 3, the model is not able to predict the modification of the wave field at the front and side of the array. Source: [25]. calculated using BEM. In principle, this approach would solve many of the previously mentioned issues.

Some preliminary work in this direction has been reported in Ref. [26]: the free surface elevation obtained using a hybrid approach was compared to direct results from WAMIT, with promising results.

\section{Current knowledge of the park effect for WEC arrays}

\subsection{Results obtained in regular waves}

\subsubsection{The q-factor}

The $q$-factor is a simple measure that quantifies the effect of wave interactions on power absorption in a WEC array. It is defined as the ratio of the power of the array to $N$ times the power from a single isolated device, $N$ being the number of units in the array.

$q(\omega)=\frac{\tilde{P}_{\text {array }}(\omega)}{N \tilde{P}_{\text {isolated }}(\omega)}$

If $q<1$, the average power per WEC in the array is less than the power of an isolated WEC. Hence, wave interactions have a destructive effect on the power absorption of the wave farm. Conversely, if $q>1$, the park effect is constructive.

This measure was introduced by Refs. [7-9] in the early 80 s. In their pioneering work, the authors of those papers showed that for several combinations of wave frequencies and array layout, it was possible to achieve a $q$-factor considerably higher than 1 (Fig. 5).

However, those are theoretical cases of optimally controlled axisymmetric heaving buoys in unidirectional regular waves. In the real world, waves are irregular, there is directional spreading, and optimal control is not realistically achievable. Consequently, in Ref. [27] the authors argue that in practice, array layouts should be optimised to minimise destructive interactions (when $q<1$ ). This is currently the prevailing view, although it is not shared by every researcher in field.

\subsubsection{Consistency conditions}

As described in Refs. [29], the $q$-factor of an optimally controlled array must satisfy the condition:

$\frac{1}{2 \pi} \int_{0}^{2 \pi} q(\omega, \beta) \mathrm{d} \beta=1$

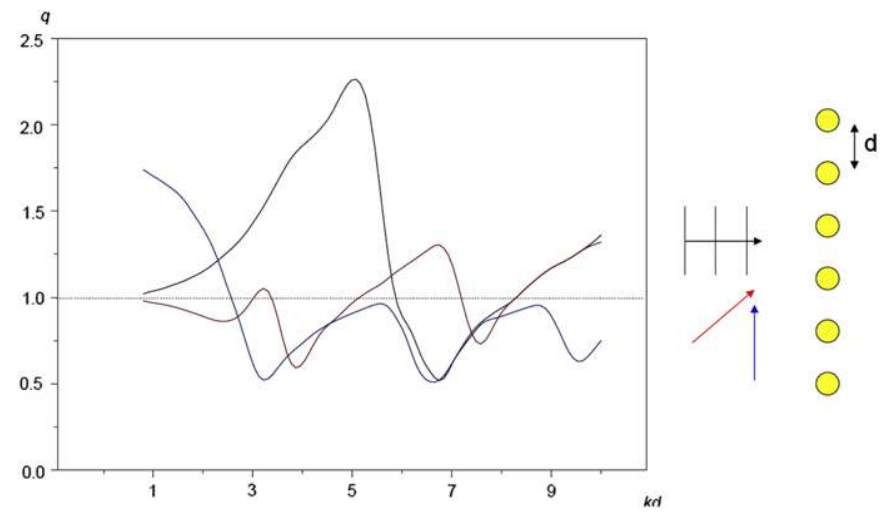

Fig. 5. $q$-factor as a function of the product of wavenumber times separation distance in a line array of optimally controlled heaving WECs, for the case of regular waves. Black, blue and red curves denote head, beam and $45^{\circ}$ incident waves. This is a classical result. (For interpretation of the references to colour in this figure legend, the reader is referred to the web version of this article.) Source: [28]. 
where $\beta$ is the direction of propagation of the incident wave.

This means that the sum of the $q$-factor over all incident wave directions is unity. In other words, if the $q$-factor is considerably greater than 1 in a particular direction, there is necessarily another direction in which it is less than 1. This is illustrated in Fig. 6 for three different arrays of five heaving WECs. One can see that the park effect is very beneficial for wave direction in the vicinity of $\beta=$ $1.5 \pi(q>1)$. However, the park effect is negative $(q<1)$ in all other directions. As explained in Refs. [28], it follows that it may be feasible to seek regions of constructive wave interactions for array placement only in cases where there is little variation in the directionality of the waves. Otherwise, it would be preferable to design the array placement in order to limit destructive effects.

Recently [30], showed a somewhat related result for the power absorption of an optimally controlled array of WECs with $N$ independent DoFs:

$\frac{1}{2 \pi} \int_{0}^{2 \pi} \tilde{P}_{\text {array }}(\omega, \beta) \mathrm{d} \beta=N \frac{J}{k}$

The sum over all directions of the power from the array $\tilde{P}_{\text {array }}$ is equal to $N$ times the wave energy flux $J$ divided by the wavenumber $k$. Therefore, to maximize the power that a device or an array of devices can extract, the number of DoFs should be as high as possible. This result indicates that wave energy devices composed of one platform supporting many independently oscillating units (such as the FO3 platform [14], the Manchester Bobber [31] or the Weptos WEC [32]) or flexible WECs (such as the Anaconda [33] or the S3 WEC [34]) might be particularly efficient at absorbing the waves.

4.1.3. Recovery rate of the wave energy flux behind an isolated WEC

In Refs. [4], the mathematical expression for the wave energy flux $J$ at distance $d$ behind a WEC is given, for the case of deep water. It is based on the asymptotic approximation for the far field component of the wave (see Section 2), and as such is valid only at sufficiently large distances. It can be written as:

$\left.J(d)=J_{0} \quad 1+\frac{2 \omega}{g} \sqrt{\frac{k}{2 \pi d}} \Im\left(\tilde{H}(0) e^{-i \frac{\pi}{4}}\right)\right)$

where $\tilde{H}(0)$ is the Kochin function [18] in the direction of incident wave propagation and $J_{0}$ is the energy flux in the incident wave.

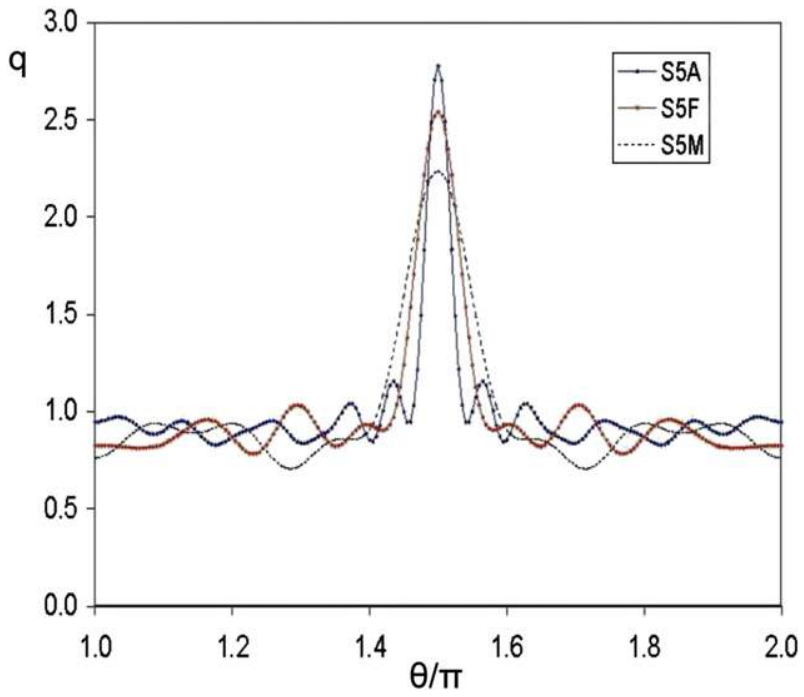

Fig. 6. $q$-factor as a function of wave incidence for an array of five heaving buoys. Buoy radius is $10 \mathrm{~m}$. The incident wave is regular with period $10 \mathrm{~s}$. Source: [29]
Therefore, one can see that the wave energy flux recovers with the square root of the distance from the source of the perturbation (the WEC). It follows that at sufficiently large distances, wave interactions between WECs can be neglected.

\subsubsection{Some additional interesting results for simple arrays for the case of regular waves}

In Refs. [3], a simple arrangement of two identical truncated cylinders is considered, with incident waves travelling parallel to the line through their centres. It is shown that due to wave interactions, the modification of the response and power absorption for the WEC at the front (the one that meets the wave first) is significantly larger than for the WEC at the back. This quite surprising result can be explained by the fact that the WEC at the front benefits from the diffracted/radiated wave originating from the WEC at the back. It illustrates the crucial importance of correctly accounting for this effect when modelling an array of WECs.

This effect is also observed in later studies by Refs. [4,5]. In the former, the same simple layout is considered, but with heaving buoys and OSWCs. The study assessed the effect of increasing distance between WECs on their interaction factors, and observed that, for distances greater than a few hundred metres, the park effect of each WEC decreased with the square root of the distance (Fig. 7). This is in agreement with the recovery rate of the wave energy flux.

It was also observed that the park effect decreased below $10 \%$ once the distance between the WECs was increased to a few hundred metres.

In Refs. [35], a mathematical model of a line array of Oscillating Water Columns is presented that investigates power performance as a function of incident wave heading and power take off. It considers the cases of optimum control and passive loading the latter being more realistic in the case of a Wells turbine. It concludes that with passive loading the park effect may be marginally beneficial, but that in most situations the absorbed energy per device is decreased.

\subsection{Results obtained in irregular waves}

\subsubsection{Important notice}

In the following, results from spectral methods are not reported. This is because, as explained in Section 3, it is believed that these methods are not able to model WEC and WEC arrays correctly.

Results for arrays of closely spaced WECs (in which the separating distance is less than a few diameters), such as the Manchester Bobber [31] or the FO3 platform [14], are not considered either. This is because it is believed that such arrays should be considered and modelled as single units, as in Ref. [14].

\subsubsection{Park effect for small arrays (up to 10 devices)}

To our knowledge, Ref. [13] reported the first investigation of the park effect for an array of WECs for the case of irregular waves. They measured yearly average power absorption. Two arrays of 5 heaving WECs, in two different layouts, were considered.

Fig. 8 shows that the device performance becomes practically independent of the spacing for separating distances greater than 4 radii (where radii ranged between 2.5 and $10 \mathrm{~m}$ ). Moreover, no significant improvement in yearly average power was obtained by individually optimising the PTO coefficient of each unit as compared to using a single value for the whole array.

Similar results were obtained:

- in Ref. [36] for an array of four heaving WECs. Absorbed power was found to be reduced by a maximum of $8 \%$ in irregular waves, and optimisation of the PTO of each individual WEC increased the absorbed power by only $1 \%$. 

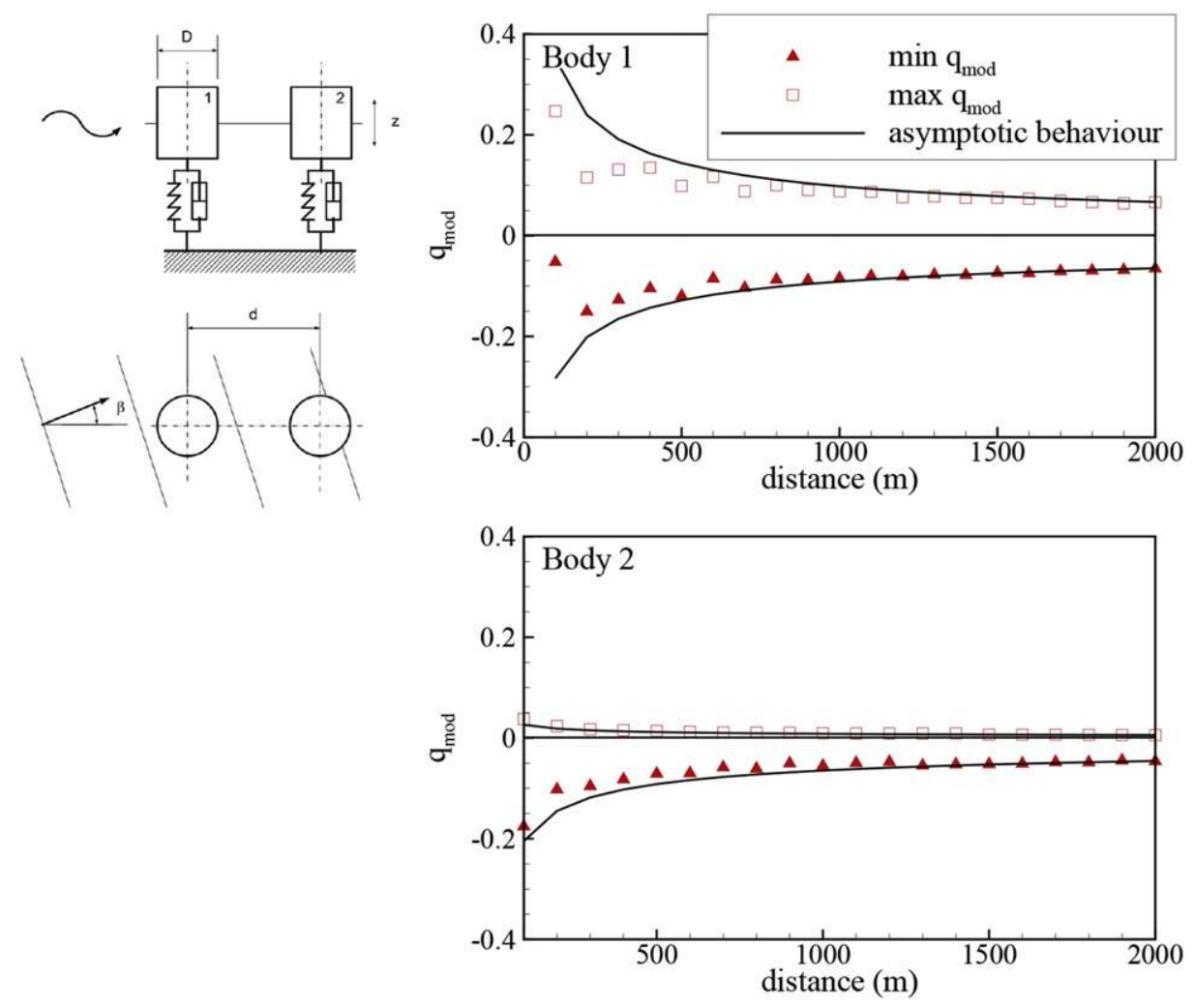

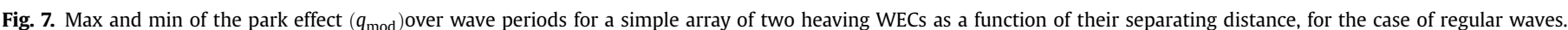

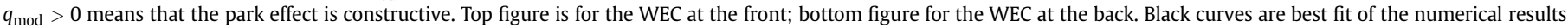
using a power law with exponent -0.5 .

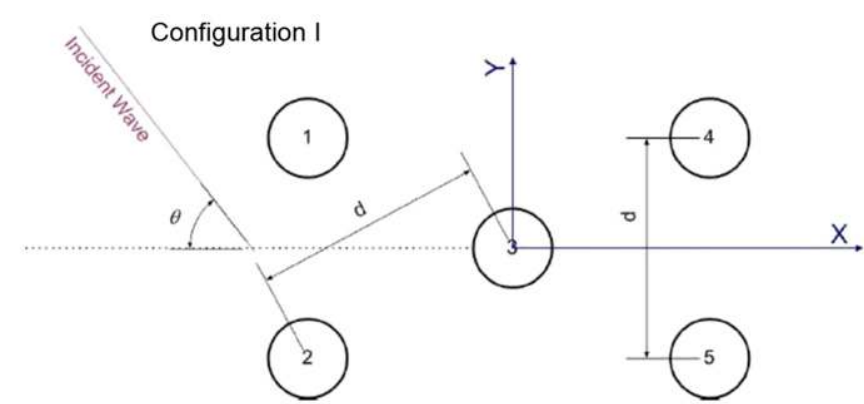

Configuration II

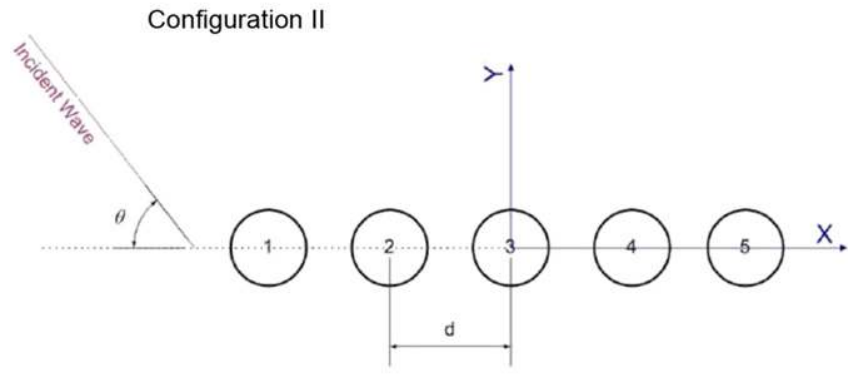

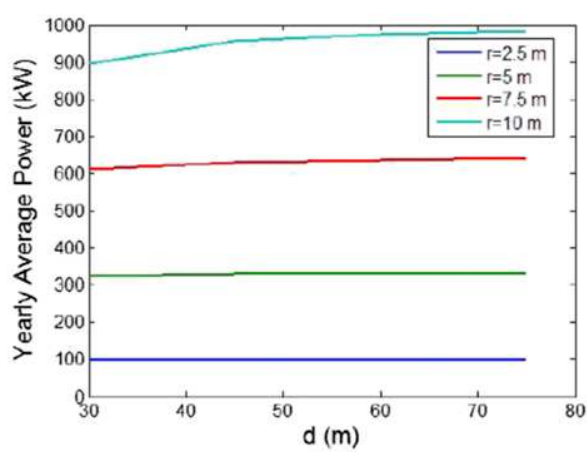

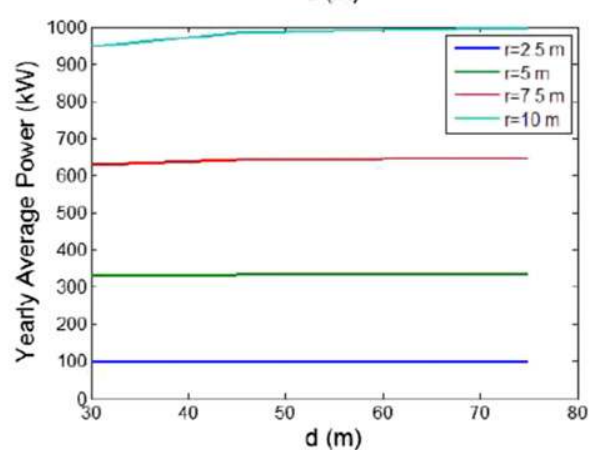

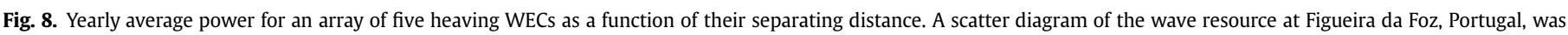
used. Source: [13]. 

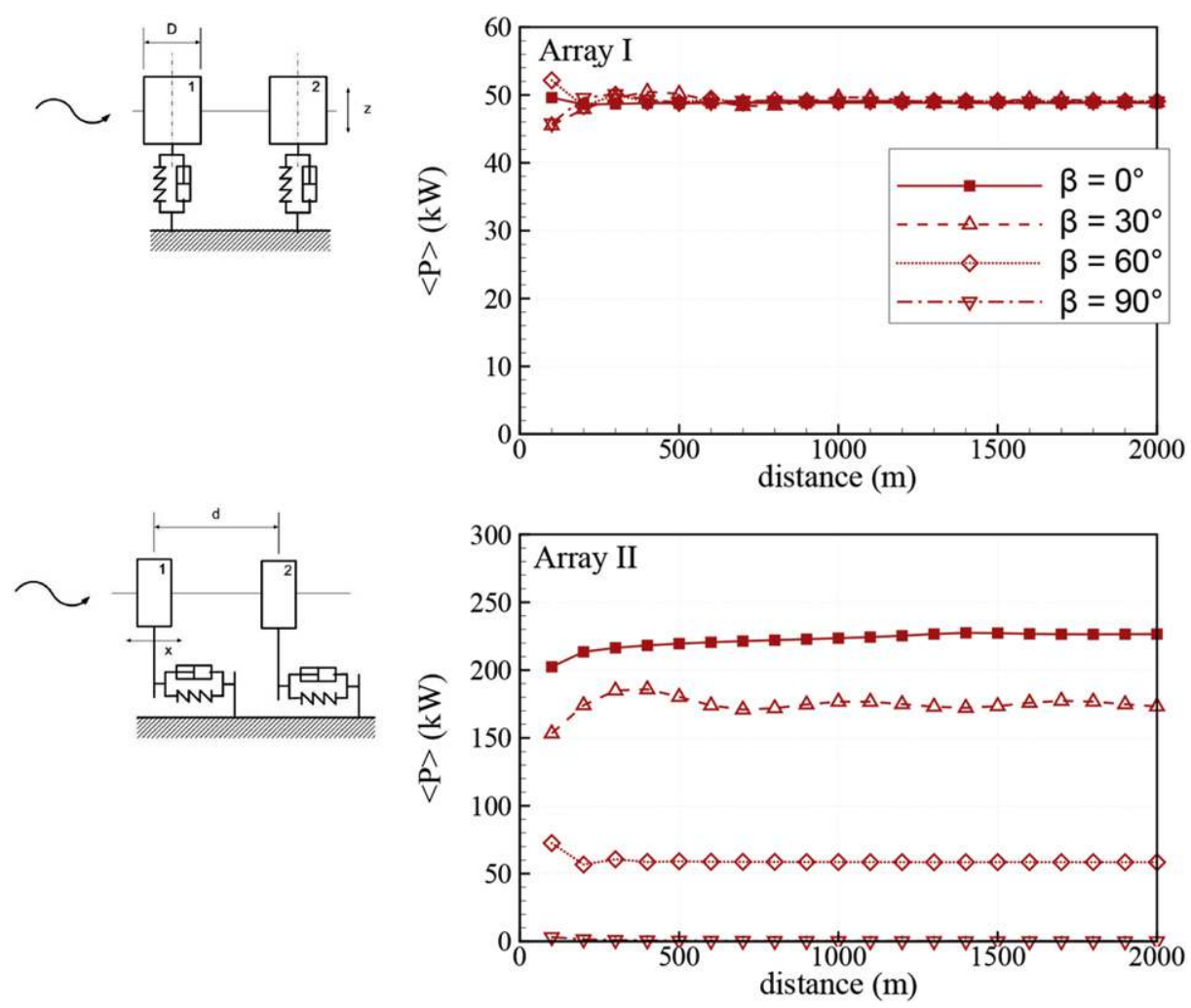

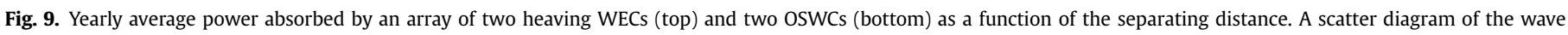
resource at a site offshore Yeu island in France was used. Source: [4]
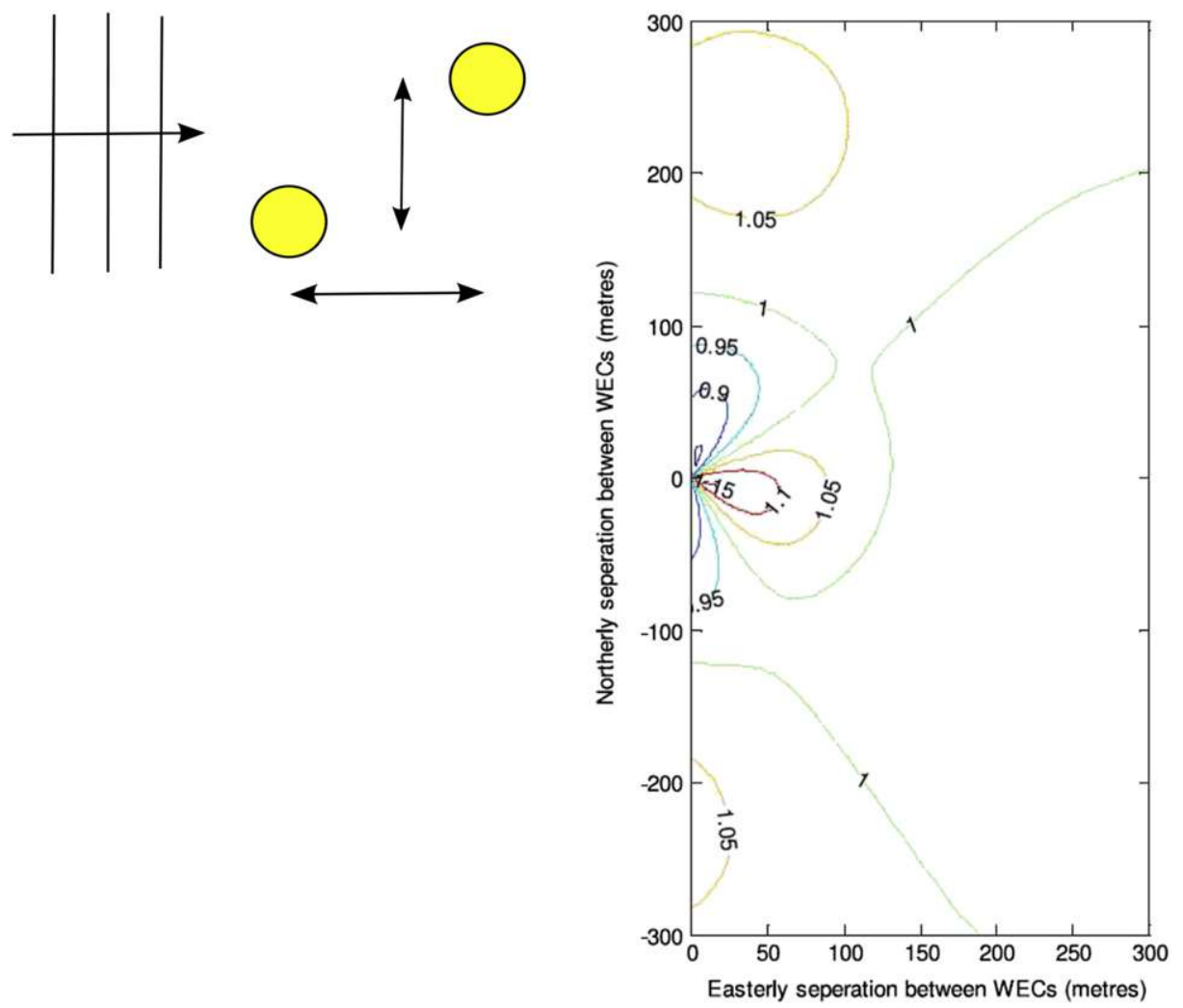

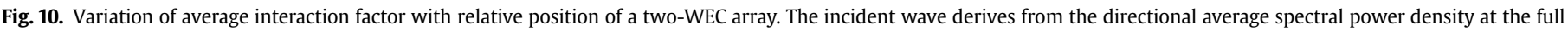
scale test site EMEC in Scotland. Source: [37]. 

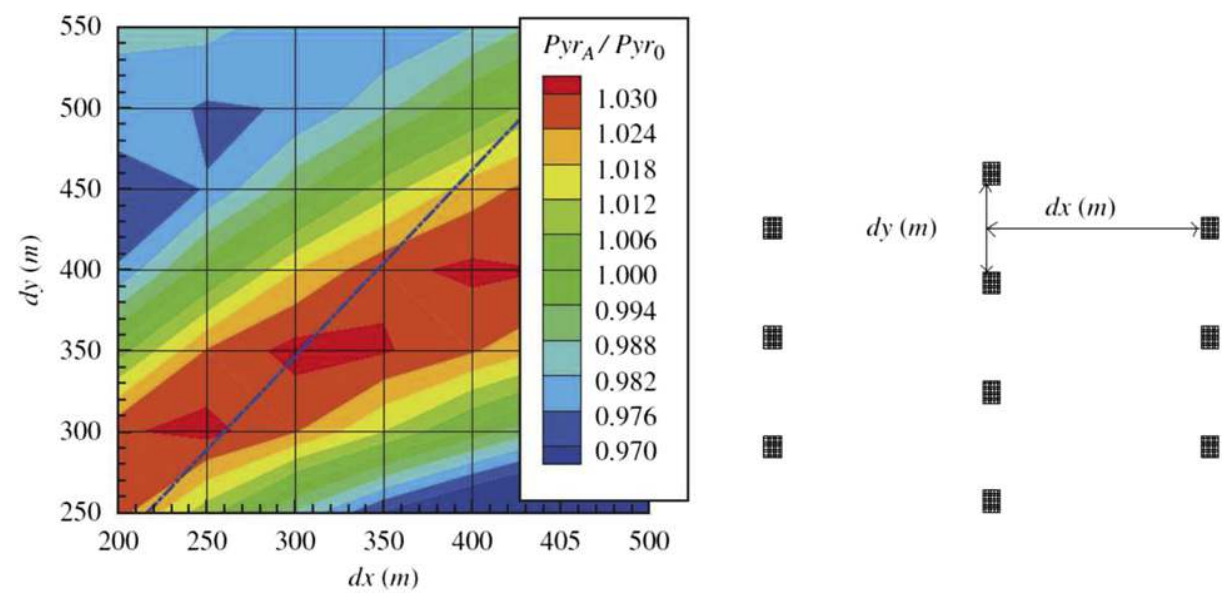

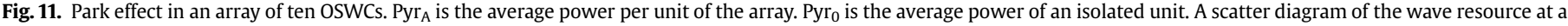
site offshore Yeu island in France was used. Source: [5].

- in Ref. [4] for an array of two heaving WECs (array I of Fig. 9), and an array of two OSWCs (array II of Fig. 9). One can see that for both arrays, the park effect is negligible once the distance is greater than a few hundred metres.

- in Refs. [37], for an array of two interacting WECs. The average interaction factor was found to be lower than $5 \%$ for separating distances larger than $100 \mathrm{~m}$ (Fig. 10).

- in Ref. [5] for an array of ten OSWCs (denoted barges in the paper). A wide range of independent longitudinal and lateral distances was tested. The yearly absorbed power of interacting devices was shown to differ by only a few percent from the yearly absorbed power of an isolated device (Fig. 11).

\subsubsection{Park effect for larger arrays (ten or more devices)}

Recently, Ref. [38] considered the case of ten heaving WECs and irregular waves. The layout for some particular sea states was optimized in order to maximize or minimize the absorbed power. Optimal layout in the maximization case led to an increase of the absorbed power by only a few percent (up to 5\%), which is in the range of the results obtained with smaller arrays in the other studies mentioned above.

However, a minimization case was also considered, in which the aim was to find the layout minimizing the absorbed power. The result was a decrease by as much as $30 \%$, which is substantial and rather unexpected.

Fig. 12 shows the corresponding layout, which is quite particular. However, overall, one can consider that the WECs are essentially located the one in front of the other: the array approximates a line parallel to the incident wave direction.

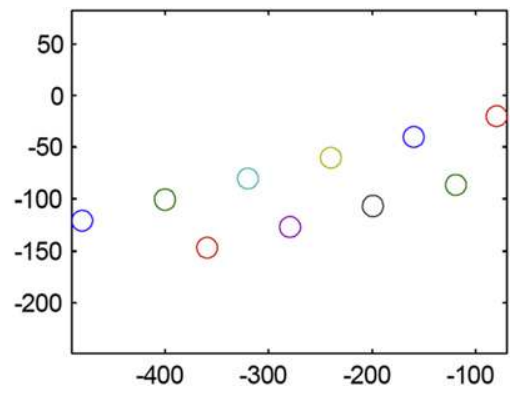

Fig. 12. Optimal layout for minimising the power absorption for an array of ten heaving WECs. The incident wave is undirectional and irregular with $\mathrm{Tp}=10.2 \mathrm{~s}$ and $\mathrm{Hs}=4 \mathrm{~m}$. The $x$ and $y$ axis are the horizontal coordinates, in meters. Source: [38]
In Refs. [5], arrays of $4 \times 4$ OSWCs (16 WECs in total) and $5 \times$ 5 OSWCs (25 WECs in total) are considered. The yearly average absorbed power per unit of a row (a row is perpendicular to the incident wave direction) reported is shown in Fig. 13. R1 corresponds to the row of WECs which meets the waves first, R4 or R5 with the WECs furthest back. The dashed black line corresponds to the yearly average power in the absence of wave interactions.

It is clear that, except for row 1, the yearly average power from row $n+1$ is less than that from row $n$. Moreover, it seems that the difference $Q$ in absorbed power between two consecutive rows is roughly the same regardless of row (except for row 1 ). Thus, one could approximately estimate the amount of power from an additional row at the back of the array as the power from the row in front of it minus the characteristic power reduction $Q$ due to the park effect. Thus, the power absorption would fall to 0 for a large number of rows. This clearly indicates that the number of rows should be as limited as possible.

\subsubsection{Impact of arrays on the surrounding wave field}

In Refs. [39], the impact of large arrays on the surrounding wave field was investigated using BEM, for the case of irregular waves with and without directional spreading. An example is shown in Fig. 14, depicting modification of the significant wave height. As expected, it appears that the wave height is reduced behind the array, and increased on its sides. However, these modifications appear to be rather small, never exceeding $10 \%$ outside the array for the unidirectional case. They are even smaller when taking into account directional spreading. Moreover, it can be observed that they decrease with increasing distance from the array. It is also reported in Ref. [39] that they are smaller for longer peak periods. Therefore, one can expect that the impact of arrays on the surrounding wave field will be limited.

\section{Tentative guidelines for the design of WEC arrays}

This review of the current literature relating to the park effet for WEC arrays leads to the following conclusions:

- Theoretically, wave interactions in an array of $N$ WECs can be constructive, thus increasing the absorbed power in comparison with $N$ isolated WECs. However, in practice (irregular waves, suboptimal control, etc.), constructive effects will be very limited and one should aim at limiting destructive interferences. 

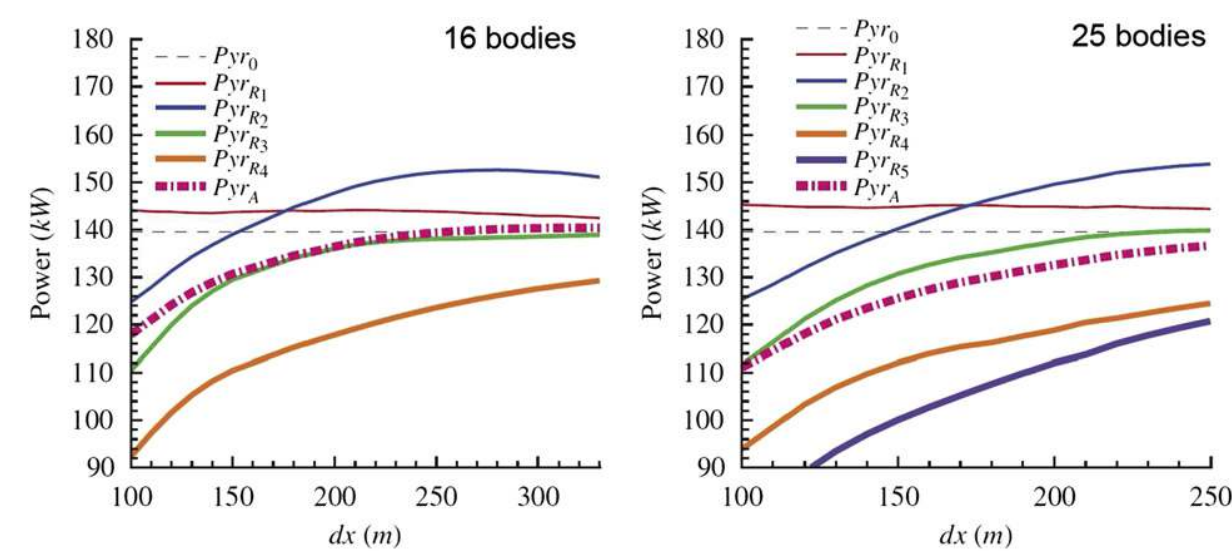

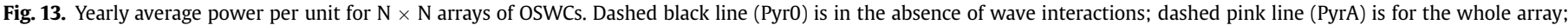

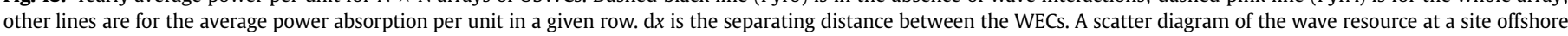
Yeu island in France was used. (For interpretation of the references to colour in this figure legend, the reader is referred to the web version of this article.) Source: [5]

- It appears that the first row (that which meets the waves first) often benefits from the waves diffracted/radiated back by the other units in the array. However, this is not usually sufficient to compensate for the decrease in power absorption of the other rows.

- In small arrays of standard layouts (fewer than ten devices on regular or shifted grids), the park effect is usually less than a few percent once the distance between the units is greater than a few hundred metres (typically $100-200 \mathrm{~m}$ for a WEC of diameter $10-20 \mathrm{~m}$ ). Taking into account the uncertainties associated with the modelling (BEM), the positioning of the WECs, the bathymetry, etc., the park effect should not be considered to be significant.

- For larger arrays (more than ten devices), it may be more important to account for the park effect. Indeed, it has been observed that the absorbed power of a given row in the array is less than the one in front, and greater than the one behind. Therefore, the number of rows should be kept as small as possible (even if the park effect was shown to be smaller than $10 \%$ in a $5 \times 5$ array of 25 OSWCs).

- For large arrays (more than ten devices), there is no indication that the number of devices per row should be limited in any manner, nor of an optimal separating distance (provided that it is sufficiently large, typically $100-200 \mathrm{~m}$ ).

- The modification of the wave field behind and around a WEC array seems to be rather small even in the case of large arrays (18 units). Moreover, it decreases with the distance from the array.

These conclusions lead to the following tentative guidelines:

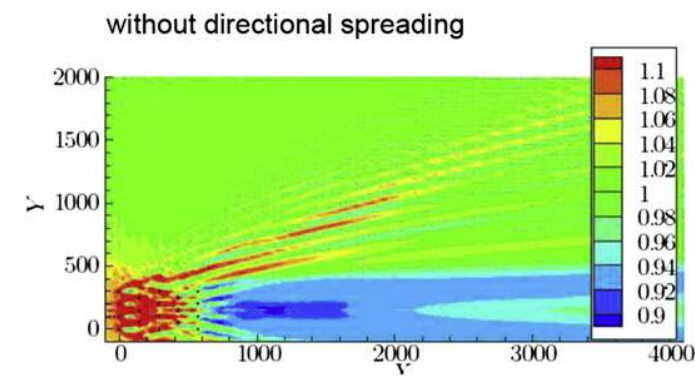

\subsection{Small conventional oscillating WEC arrays (up to ten devices of 10-20 m typical dimension)}

For small arrays (fewer than 10 devices of 10-20 m typical dimension) with standard layouts (regular or shifted grids with separating distance of 100-200 m), the park effect can be neglected: studies show that it usually accounts for less than a few percent of the mean annual power.

\subsection{Larger arrays of conventional WECs (more than 10 ten devices of 10-20 m typical dimension)}

For larger arrays (more than ten devices), the park effect might be increasingly important as the number of rows (lines of WECs perpendicular to the incident wave direction) increases. Therefore, the number of rows should be kept as small as possible, with a separating distance as large as possible. As an indication, the park effect was found to be $5 \%$ in an array of 25 OSWCs with separating distance $200 \mathrm{~m}$, with a decrease of $20 \%$ in mean annual power absorption for the fifth row in the array. There is no indication that the number of devices in a row should be limited in any manner, nor that there is an optimum lateral separating distance (provided it is a least 100$200 \mathrm{~m}$ ). Finally, it should be expected that the front row of the array may demonstrate greater power absorption than isolated devices.

\subsection{Arrays of non-conventional oscillating WECs (WECs of typical dimension much greater than 10-20 m)}

For arrays of non-conventional WECs (WECs of typical dimension much greater than $10-20 \mathrm{~m}$ ), there is no information available.

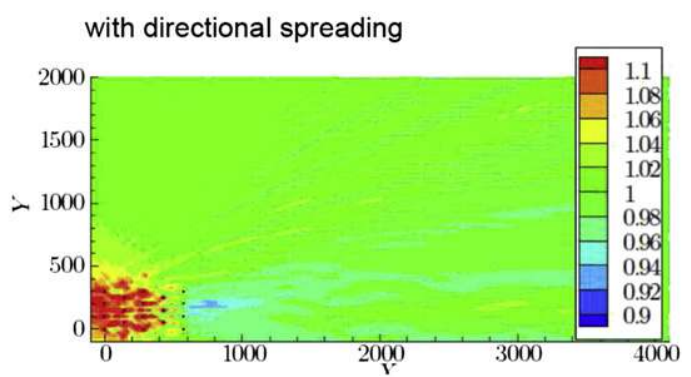

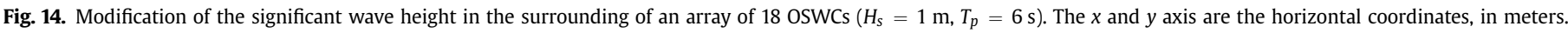
Source: [39] 
However, similar trends to the previous cases can be expected provided that aspect ratio is maintained:

- For small arrays (fewer than ten devices of typical dimension $B$ ), with standard layouts and separating distance $10 B$ to $20 B$, the park effect should be negligible.

- For larger arrays (more than ten devices), the number of rows should be kept as small as possible, with separating distance as great as possible $(10 B-20 B)$.

\subsection{Impact of WEC arrays on the surrounding wave field}

The modification of the wave field behind and around an array seems to be rather small even in the case of large arrays (only a few percent of significant height). Moreover, it decreases with the distance from the array. Therefore, the impact on the wave climate by small WEC arrays is unlikely to be significant. This is the focus of a future investigation by the authors.

\section{Conclusion}

This paper reviewed the current literature relating to the park effect for arrays of WECs, and revealed consistent trends in terms of the significance of this effect as a function of the number of WECs in the array and the separating distance between them. Therefore, tentative conclusions and guidelines have been drawn regarding the design of WEC arrays in order to mitigate the park effect.

Since most of the published work deals with rather small arrays of rather small devices, confidence in the guidelines is high for these cases. However, in the case of larger arrays, there have been only a few published studies to date. Thus, the degree of confidence is much lower and further research would be worthwhile. Here, the difficulty lies in the limitations of the available numerical tools.

For BEM codes, the limitations are practical: memory and CPU requirements increase rapidly with the number and size of WECs in the array. For Boussinesq and spectral wave models, the limitation is theoretical and relates to how to correctly account for a WEC in the model. These two issues will need to be overcome before analysis of large scale WEC arrays is feasible.

\section{Acknowledgements}

The author gratefully acknowledges the financial support from the European Commission through the 7th Framework Programme (MARINA Platform - Marine Renewable Integrated Application Platform, Grant Agreement 241402) which made this work possible.

\section{References}

[1] Falnes J. A review of wave energy extraction. Marine Structures 2007;vol. 20: 185-201.

[2] Wypych M, Le-Ngoc L, Alexander K, Gardner A. On the application of circularcylindrical waves to ocean power absorption. Ocean Engineering 2012;vol. 40: 69-75.

[3] Child B, Venugopal V. Interaction of waves with an array of floating wave energy devices. In: Proc. of the 7th European wave and tidal energy conference, Porto, Portugal; 2007.

[4] Babarit A. Impact of long separating distances on the energy production of two interacting wave energy converters. Ocean Engineering 2010;vol. 37: 718-29.

[5] Borgarino B, Babarit A, Ferrant P. Impact of wave interactions effect on energy absorption in large arrays of wave energy converters. Ocean Engineering 2011;vol. 41:79-88.

[6] Folley M, Babarit A, Child B, Forehand D, O’Boyle L, Silverthorne K, et al. A review of numerical modelling of wave energy converters arrays. In: Proc. of the 31st international conference on ocean, offshore and artic engineering, Rio de Janeiro, Brazil; 2012.

[7] Budal K. Theory of absorption of wave power by a system of interacting bodies. Journal of Ship Research 1977;vol. 21:248-53.

[8] Evans DV. Some theoretical aspects of three dimensional wave energy absorbers. In: Proc. of the 1st symposium on wave energy utilization, Gothenburg, Sweden; 1979.

[9] Falnes J. Radiation impedance matrix and optimum power absorption for interacting oscillators in surface waves. Applied Ocean Research 1980;vol. 2: 75-80.

[10] Garnaud X, Mei CC. Bragg scattering and wave-power extraction by an array of small buoys. Proc of the Royal Society A: Mathematical, Physical and Engineering Sciences 2009;vol. 466:79-106.

[11] Mavrakos S, McIver P. Comparison of methods for computing hydrodynamic characteristics of arrays of wave power devices. Applied Ocean Research 1997; vol. 19:283-91.

[12] Justino PAP, Clément AH. Hydrodynamic performance for small arrays of submerger spheres. In: Proc. of the 5th European wave and tidal energy conference, Cork, Ireland; 2003.

[13] Ricci P, Saulnier JB, de O. Falcão AF. Point-absorber arrays: a configuration study off the Portuguese West-Coast. In: Proc. of the 7th European wave and tidal energy conference, Porto, Portugal; 2007.

[14] De Backer G, Vantorre M, Beels C, De Rouck J, Frigaard P. Power absorption by closely spaced point absorbers. IET Renewable Power Generation 2010; vol. 4(6):579-91.

[15] Taghipour R, Efficient prediction of dynamic response for flexible and multibody marine structures, PhD Thesis, center for ships and ocean structures, NTNU Trondheim, Norway; 2008

[16] Vicente PC, de O. Falcão AF, Gato LMC, Justino PAP. Dynamics of arrays of floating-point absorber wave energy converters with inter-body and bottom slack-mooring connections. Applied Ocean Research 2009;vol. 31: 267-81.

[17] Borgarino B, Babarit A, Ferrant P. An implementation of the fast multipole algorithm for wave interaction problems on sparse arrays of floating bodies. Journal of Engineering Mathematics 2012:1-18.

[18] Mei CC, Stiassnie M, Yue DKP. Theory and applications of ocean surface waves. Part 1, Linear aspects. Singapore: World Scientific; 2005.

[19] Simon MJ. Multiple scattering in arrays of axisymmetric wave energy devices - a matrix method using a plane wave approximation. Journal of Fluid Mechanics 1982; vol. 120:1-25.

[20] Millar DL, Smith HCM, Reeve DE. Modelling analysis of the sensitivity of shoreline change to a wave farm. Ocean Engineering 2007;vol. 34: 884-901.

[21] Venugopal V, Smith GH. Wave climate investigation for an array of wave power devices. In: Proc. of the 7th European wave and tidal energy conference, Porto, Portugal; 2007.

[22] Vidal C, Mendez FJ, Diaz G, Legaz L. Impact of Santoa WEC installation on the littoral process. In: Proc. of the 7th European wave and tidal energy conference, Porto, Portugal; 2007.

[23] Mendes L, Palha A, Fortes CJ, Brito-Melo A, Sarmento A. Analysis of the impact of a pilot zone for wave energy conversion offshore portugal. In: Proc. of the 18th international offshore and polar engineering conference; 2008. p. $401-8$.

[24] Alexandre A, Stallard T, Stansby PK. Transformation of wave spectra across a line of wave devices. In: Proc. of the 8th European wave and tidal energy conference, Uppsala, Sweden; 2009.

[25] Silverthorne K, Folley M. A new numerical representation of wave energy converters in a spectral wave model. In: Proc. of the 9th European wave and tidal energy conference, Southampton, The UK; 2011.

[26] Beels C. Optimization of the lay-out of a farm of wave energy converters in the North Sea. Analysis of wave power resources, wake effects, production and cost. PhD thesis, Gent University, Belgium; 2010.

[27] Thomas G, Evans DV. Arrays of three dimensional wave energy absorbers. Journal of Fluid Mechanics 1981;vol. 108:67-88.

[28] Thomas G. Some observations on modelling arrays of wavepower devices. In: Slideshow of presentation at NUIM wave energy workshop, Maynooth, Ireland, October 25th; 2011.

[29] Fitzgerald C, Thomas G. A preliminary study on the optimal formation of an array of wave power devices. In: Proc. of the 7th European wave and tidal energy conference, Porto, Portugal; 2007.

[30] Wolgamot HA, Taylor PH, Eatock Taylor R. The interaction factor and directionality in wave energy arrays. Ocean Engineering 2012;vol. 47: 65-73.

[31] Weller S, Stallard T, Stansby PK. Experimental measurements of irregular wave interaction factors in closely spaced arrays. In: Proc. of the 8th European wave and tidal energy conference, Uppsala, Sweden; 2009.

[32] Pecher A, Kofoed JP, Larsen T. Design specifications for the WEPTOS Hanstholm WEC. Energies 2012; vol. 5:1001-17.

[33] Farley FJM, Rainey RCT, Chaplin JR. Rubber tubes in the sea. Philosophical Transactions of the Royal Society A 2012;vol. 370:381-402.

[34] Jean P, Wattez A, Ardoise G, Melis C, Van Kessel R, Fourmon A, et al. Standing wave tube electro active polymer wave energy converter. In: Proc. of SPIE8340, 83400C; 2012

[35] de O. Falcão AF. Wave power absorption by a periodic linear array of oscillating water columns. Ocean Engineering 2002;vol. 29(11):1163-86. 
[36] Cruz J, Sykes R, Siddorn P, Eatock Taylor R. Wave farm design: preliminary studies on the influences of wave climate, array layout and farm control. In: Proc. of the 8th European wave and tidal energy conference, Uppsala, Sweden; 2009.

[37] Folley M, Whittaker TJT. The effect of sub-optimal control on the spectral wave climate on the performance of wave energy converter arrays. Applied Ocean Research 2009;vol. 31(4):260-6.
[38] Child BFM, Cruz J, Livingstone M. Development of a tool for optimising arrays of wave energy converters. In: Proc. of the 9th European wave and tidal energy conference, Southampton, The UK; 2011.

[39] Borgarino B, Babarit A, Ferrant P. Impact of the separating distance between interacting wave energy converters on the overall energy extraction of an array. In: Proc. of the 9th European wave and tidal energy conference, Southampton, The UK; 2011. 